\title{
The Quasi-Linear Operator Outer Generalized Inverse with Prescribed Range and Kernel in Banach Spaces
}

\author{
Jianbing $\mathrm{Cao}^{1,2}$ and Yifeng $\mathrm{Xue}^{2}$ \\ ${ }^{1}$ Department of Mathematics, Henan Institute of Science and Technology, Xinxiang, Henan 453003, China \\ ${ }^{2}$ Department of Mathematics, East China Normal University, Shanghai 200241, China
}

Correspondence should be addressed to Yifeng Xue; yfxue@math.ecnu.edu.cn

Received 20 January 2013; Accepted 4 July 2013

Academic Editor: C. Morales

Copyright (c) 2013 J. Cao and Y. Xue. This is an open access article distributed under the Creative Commons Attribution License, which permits unrestricted use, distribution, and reproduction in any medium, provided the original work is properly cited.

Let $X$ and $Y$ be Banach spaces, and let $A: X \rightarrow Y$ be a bounded linear operator. In this paper, we first define and characterize the quasi-linear operator (resp., out) generalized inverse $A_{T, S}^{(h)}$ (resp., $A_{T, S}^{(2, h)}$ ) for the operator $A$, where $T \subset X$ and $S \subset Y$ are homogeneous subsets. Then, we further investigate the perturbation problems of the generalized inverses $A_{T, S}^{(h)}$ and $A_{T, S}^{(2, h)}$. The results obtained in this paper extend some well-known results for linear operator generalized inverses with prescribed range and kernel.

\section{Introduction and Preliminaries}

Let $X$ and $Y$ be Banach spaces, let $T: X \rightarrow Y$ be a mapping, and let $D \subset X$ be a subset of $X$. Recall from $[1,2]$ that a subset $D$ in $X$ is called to be homogeneous if for any $x \in$ $D$ and $\lambda \in \mathbb{R}$, we have $\lambda x \in D$. If for any $x \in \mathscr{D}(T)$ and $\lambda \in \mathbb{R}$, we have $T(\lambda x)=\lambda T(x)$, then we call $T$ as a homogeneous operator on $\mathscr{D}(T)$, where $\mathscr{D}(T)$ is the domain of $T$; $T$ is called a bounded homogeneous operator if $T$ maps every bounded set in $\mathscr{D}(T)$ into bounded set in $Y$. Denote by $H(X, Y)$ the set of all bounded homogeneous operators from $X$ to $Y$. Equipped with the usual linear operations for $H(X, Y)$, and for $T \in H(X, Y)$, the norm is defined by $\|T\|=$ $\sup \{\|T x\| \mid\|x\|=1, x \in X\}$, and then similar to the space $B(X, Y)$ of all bounded linear operators from $X$ to $Y$, we can easily prove that $(H(X, Y),\|\cdot\|)$ is a Banach space (cf. $[2,3])$. Throughout this paper, we denote by $\mathscr{D}(T), \mathcal{N}(T)$, and $\mathscr{R}(T)$ the domain, the null space, and the range of a bounded homogeneous operator $T \in H(X, Y)$, respectively. Obviously, we have $B(X, Y) \subset H(X, Y)$.

For an operator $A \in B(X, Y)$, let $T$ and $S$ be closed subspaces of $X$ and $Y$, respectively. Recall that the out inverse $A_{T, S}^{(2)}$ with prescribed range $T$ and kernel $S$ is the unique operator $G \in B(Y, X)$ satisfying $B A B=B, \mathscr{R}(B)=T, \mathcal{N}(B)=S$. It is well known that the important kinds of generalized inverses, the Moore-Penrose inverse, the Drazin inverse, the group inverse, and so on, are all generalized inverse $A_{T, S}^{(2)}$ (cf. $[4,5])$. Researches on the generalized inverse $A_{T, S}^{(2)}$ of operators or matrices have been actively ongoing for many years (see [5-12], e. g.).

Let $A \in B(X, Y)$ and let $T$ and $S$ be two homogeneous subsets in $X$ and $Y$, respectively. Motivated by related work on $A_{T, S}^{(2)}$ in the literature mentioned above and by our own recent research papers $[13,14]$, in this paper, we will establish the definition of the quasi-linear operator outer generalized inverse $A_{T, S}^{(2, h)}$ with prescribed range $T$ and kernel $S$. We give the necessary and sufficient conditions for the existence of the generalized inverses $A_{T, S}^{(2, h)}$, and we will also study the perturbation problems of the generalized inverse $A_{T, S}^{(2, h)}$. Similar results on the generalized inverse $A_{T, S}^{(h)}$ are also given.

\section{Definitions and Some Characterizations of $A_{T, S}^{(2, h)}$ and $A_{T, S}^{(h)}$}

We first give the concepts of quasi-additivity and quasi-linear projectors in Banach spaces, which are important for us to present the main results in this paper.

Definition 1. Let $M$ be a subset of $X$ and let $T: X \rightarrow Y$ be a mapping. Ones calls $T$ as quasi-additive on $M$ if $T$ satisfies

$$
T(x+z)=T(x)+T(z), \quad \forall x \in X, \forall z \in M .
$$


Definition 2 (see $[3,15])$. Let $X$ be a Banach space. A mapping $P: X \rightarrow X$ is called a quasi-linear projector on $X$, if $P$ satisfies the following conditions:

(1) $P$ is a homogeneous operator;

(2) $P$ is idempotent; that is, $P^{2}=P$;

(3) $P$ is quasi-additive on $\mathscr{R}(P)$; that is, for any $x \in X$ and any $z \in \mathscr{R}(P)$, one has

$$
P(x+z)=P(x)+P(z)=P(x)+z .
$$

If the mapping $P$ only satisfies the conditions (1) and (2) above, then we call $P$ as a homogeneous projector.

From Definition 2, we see that the quasi-linear projectors include all the usually used projectors, such as the linear projectors in linear spaces, the bounded linear projectors, the metric projectors in Banach spaces, and the orthogonal projectors in Hilbert spaces (cf. $[3,15,16])$. We have the following important property for quasi-linear projector.

Lemma 3 (see [15, Lemma 2.5]). If $P \in H(X, X)$ is a bounded quasi-linear projector on $X$, then the range $\mathscr{R}(P)$ of $P$ is a closed linear subspace of $X$.

We should note that, although the range $\mathscr{R}(P)$ of a bounded quasi-linear projector $P$ is a closed linear subspace, but in general, the kernel $\mathcal{N}(P)$ of a bounded quasi-linear projector $P$ is not a (closed) subspace. Let $P \in H(X)$ be a quasi-linear projector. Then by Lemma $3, \mathscr{R}(P)$ is a closed linear subspace of $X$ and $\mathscr{R}(I-P)=\mathscr{N}(P)$. Thus, we can define "the quasi-linearly complement" of a closed linear subspace as follows. Let $V$ be a closed subspace of $X$. If there exists a bounded quasi-linear projector $P$ on $X$ such that $V=\mathscr{R}(P)$, then $V$ is said to be bounded quasi-linearly complemented in $X$ and $\mathcal{N}(P)$ is the bounded quasi-linear complement of $V$ in $X$. In this case, as usual, we may write $X=$ $V \dot{+} \mathcal{N}(P)$, where $\mathcal{N}(P)$ is a homogeneous subset of $X$ and " $\dot{+}$ " means that $V \cap \mathscr{N}(P)=\{0\}$ and $X=V+\mathcal{N}(P)$.

Now we establish the definitions of some types of homogeneous (or quasi-linear) operator generalized inverses with prescribed range and kernel as follows.

Definition 4. Let $A \in B(X, Y)$. Let $T$ and $S$ be homogeneous subsets in $X$ and $Y$, respectively. Let $B \in H(Y, X)$ be a bounded homogeneous operator. Consider the following equations:

$$
\begin{aligned}
\text { (1) } A B A=A, & \text { (2) } B A B=B, \\
\text { (3) } \mathscr{R}(B)=T, & \text { (4) } \mathcal{N}(B)=S .
\end{aligned}
$$

(i) If $B \in H(Y, X)$ satisfies (2)-(4) in (3), then the operator $B$ is called to be the homogeneous outer generalized inverse of $A$ with prescribed range $T$ and kernel $S$. We denoted it by $A_{T, S}^{(2, H)}$.

(ii) If $B \in H(Y, X)$ satisfies all equations in (3), then the operator $B$ is called to be the homogeneous generalized inverse of $A$ with prescribed range $T$ and kernel $S$. We denoted it by $A_{T, S}^{(H)}$. (iii) If $B \in H(Y, X)$ is quasi-additive on $A T$ and satisfies (2)-(4) in (3), then the operator $B$ is called to be the quasi-linear outer generalized inverse of $A$ with prescribed range $T$ and kernel $S$. We denoted it by $A_{T, S}^{(2, h)}$.

(iv) If $B \in H(Y, X)$ is quasi-additive on $\mathscr{R}(A)$ and satisfies all equations in (3), then the operator $B$ is called to be the quasi-linear generalized inverse of $A$ with prescribed range $T$ and kernel $S$. We denoted it by $A_{T, S}^{(h)}$.

Remark 5. Let $A \in B(X, Y)$. Assume that $\mathscr{N}(A)$ and $\mathscr{R}(A)$ are Chebyshev subspaces in $X$ and $Y$, respectively. Then the Moore-Penrose metric generalized inverse $A^{M}$ of $A$ uniquely exists (cf. $[3,17,18])$. Let

$$
S=F_{Y}^{-1}\left(\mathscr{R}(A)^{\perp}\right), \quad T=F_{X}^{-1}\left((\mathcal{N}(A))^{\perp}\right),
$$

where $F_{X}: X \rightarrow X^{*}$ is the set-valued dual mapping of $X, \mathscr{R}(A)^{\perp}=\left\{f \in X^{*} \mid f(x)=0, \forall x \in \mathscr{R}(A)\right\}$ and $F_{X}^{-1}\left(\mathscr{R}(A)^{\perp}\right)=\left\{x \in X \mid F_{X}(x) \cap \mathscr{R}(A)^{\perp} \neq \emptyset\right\}$. Then $S$ and $T$ are homogeneous subsets in $X$ and $Y$, respectively, and

$$
\begin{array}{cc}
A^{M} A A^{M}=A, & A^{M} A A^{M}=A^{M}, \\
\mathscr{R}\left(A^{M}\right)=T, & \mathcal{N}\left(A^{M}\right)=S .
\end{array}
$$

In this case, we have $A^{M}=A_{T, S}^{(H)}$. Furthermore, if $A^{M}$ is quasi-additive on $\mathscr{R}(A)$, then $A^{M}=A_{T, S}^{(h)}$. Thus, it is meaningful to study the quasi-linear operator (outer) generalized inverses with prescribed range and kernel.

In this paper, we mainly study the generalized inverses $A_{T, S}^{(2, h)}$ and $A_{T, S}^{(h)}$. Motivated by the properties of linear operator generalized inverses with prescribed range and kernel, in the rest of this section, we will give some characterizations of the generalized inverses $A_{T, S}^{(2, h)}$ and $A_{T, S}^{(h)}$.

Theorem 6. Let $A \in B(X, Y)$. Let $T$ and $S$ be homogeneous subsets in $X$ and $Y$, respectively. Then the following statements are equivalent:

(1) there exists some $B \in H(Y, X)$ such that $B$ is quasiadditive on $A T$ and $B A B=B, \mathscr{R}(B)=T, \mathcal{N}(B)=S$;

(2) $\mathcal{N}(A) \cap T=\{0\}, Y=A T+S$ and $T \subset X$ is a closed linear subspace.

Proof. (1) $\Rightarrow$ (2) Suppose that (1) holds. Put $P=B A$ and $Q=$ $A B$. Since $B A B=B$, we have $P=P^{2}$ and $\mathscr{R}(P)=\mathscr{R}(B A)=$ $\mathscr{R}(B)=T$. In order to prove $T \subset X$ is a closed linear subspace, by Lemma 3, we only need to show that $P$ is a bounded quasi-linear projector. Let $x \in X$ and $z \in T=\mathscr{R}(B A)$, we get $z=B A z$ and $A B A z \in A T$. Noting that $B$ is quasi-additive on $A T$, then

$$
\begin{aligned}
P(x+z) & =B A(x+B A z)=B(A x+A B A z) \\
& =B A x+B A B A z \\
& =P x+z .
\end{aligned}
$$


Therefore, $P$ is a bounded quasi-linear projector and then $T$ is a closed linear subspace in $X$. Now we show that $\mathcal{N}(A) \cap$ $T=\{0\}$. Let $x \in \mathscr{N}(A) \cap T$. Since $\mathscr{R}(B)=T$, then $A x=0$ and $x=B w$ for some $w \in Y$. So $x=B w=B A B w=B A x=0$. Therefore, we have $\mathcal{N}(A) \cap T=\{0\}$. Next we will show that $Y=A T+S$. For any $y \in Y$, we have $y=\left(I_{Y}-A B\right) y+A B y$. Since we have proved that $T$ is a closed linear subspace of $X$, we have $A B y \in \mathscr{R}(Q)=\mathscr{R}(A B)=A \mathscr{R}(B)=A T$. Also noting that $B$ is quasi-additive on $A T$ and $B A B=B$, we get $\left(I_{Y}-A B\right) y \in \mathcal{N}(B)=S$. Thus, we get $Y=A T+S$. From $B A B=$ $B$, we can also get $Q=Q^{2}$ and $S=\mathcal{N}(B)=\mathcal{N}(A B)=\mathcal{N}(Q)$; therefore, $A T \cap S=\emptyset$ and then $Y=A T+S$.

(2) $\Rightarrow$ (1) Suppose that (2) holds. Since $\mathcal{N}(A) \cap T=\{0\}$ and $T$ is a closed linear subspace, we see $\left.A\right|_{T}: T \rightarrow A T$ is well defined, which is also a linear bijective mapping, so $\left.A\right|_{T}$ has a linear inverse $\left(\left.A\right|_{T}\right)^{-1}: A T \rightarrow T$. Since $Y=A T \dot{+} S$, then for any $y \in Y$, it can be uniquely written as $y=y_{1}+y_{2}$ with $y_{1} \in$ $A T$ and $y_{2} \in S$. Now we can define a mapping $B: Y \rightarrow X$ by $B y=\left(\left.A\right|_{T}\right)^{-1} y_{1}$. It is easy to see that $B A B=B, \mathscr{R}(B)=T$ and $\mathcal{N}(B)=S$ by $Y=A T+S$. Since $\mathcal{N}(B)=S$ is a homogeneous subsets in $Y$, we see $B \in H(Y, X)$ is a homogeneous operator. Now we need to prove $B$ is quasi-additive on $A T$. In fact, for any $y \in Y$ and $z \in A T$, since $Y=A T+S$, we can write $y$ as $y=y_{1}+y_{2}$, where $y_{1} \in A T$ and $y_{2} \in S$. Noting that $y_{1}+z \in$ $A T$ since $A T \subset X$ is a linear subspace, then we can check that

$$
\begin{aligned}
B(y+z) & =B\left(y_{1}+y_{2}+z\right)=B\left(\left(y_{1}+z\right)+y_{2}\right) \\
& =\left(\left.A\right|_{T}\right)^{-1}\left(y_{1}+z\right)=\left(\left.A\right|_{T}\right)^{-1} y_{1}+\left(\left.A\right|_{T}\right)^{-1} z \\
& =B y+B z .
\end{aligned}
$$

Therefore, $B$ is quasi-additive on $A T$.

Theorem 7. Let $A \in B(X, Y)$. Let $T$ and $S$ be homogeneous subsets in $X$ and $Y$, respectively. Then the following statements are equivalent.

(1) There exists some $B \in H(Y, X)$ such that $B$ is quasiadditive on $\mathscr{R}(A)$ and

$$
\begin{array}{ll}
A B A=A, & B A B=B, \\
\mathscr{R}(B)=T, & \mathcal{N}(B)=S .
\end{array}
$$

(2) $X=\mathcal{N}(A) \dot{+} T, Y=\mathscr{R}(A)+S$ and $T \subset X$ is a closed linear subspace.

(3) $Y=A T+S, \mathcal{N}(A) \cap T=\{0\}, \mathscr{R}(A) \cap S=\{0\}$ and $T \subset X$ is a closed linear subspace.

Proof. (1) $\Rightarrow$ (2) Suppose that (1) holds, Put $P=B A$ and $Q=$ $A B$. Similar to the proof of Theorem 6, we have $P=P^{2}, Q=$ $Q^{2}$ and $T \subset X$ is a closed linear subspace. Note that we also have the following relation:

$$
\begin{aligned}
& \mathscr{R}(B A) \subset \mathscr{R}(B)=\mathscr{R}(B A B) \subset \mathscr{R}(B A), \\
& \mathscr{R}(A B) \subset \mathscr{R}(A)=\mathscr{R}(A B A) \subset \mathscr{R}(A B), \\
& \mathscr{N}(B A) \subset \mathscr{N}(A B A)=\mathscr{N}(A) \subset \mathscr{N}(B A), \\
& \mathcal{N}(B) \subset \mathscr{N}(A B) \subset \mathscr{N}(B A B)=\mathscr{N}(B) .
\end{aligned}
$$

Thus, we get that

$$
\begin{gathered}
\mathscr{R}(P)=\mathscr{R}(B A)=\mathscr{R}(B)=T, \\
\mathscr{R}(Q)=\mathscr{R}(A B)=\mathscr{R}(A), \\
\mathcal{N}(P)=\mathscr{N}(B A)=\mathscr{N}(A), \\
\mathcal{N}(Q)=\mathscr{N}(A B)=\mathscr{N}(B)=S .
\end{gathered}
$$

From the above equations, we can obtain $X=\mathcal{N}(A)+T$ and $Y=\mathscr{R}(A)+S$.

(2) $\Rightarrow$ (3) If $X=\mathscr{N}(A)+T$ and $Y=\mathscr{R}(A)+S$, then it is obvious that $\mathscr{N}(A) \cap T=\{0\}, \mathscr{R}(A) \cap S=\{0\}$. We only need to show $A T=\mathscr{R}(A)$. Obviously, we have $A T \subset \mathscr{R}(A)$. Now for any $y \in \mathscr{R}(A)$, we have $y=A x$ for some $x \in X$. Since $X=$ $\mathcal{N}(A) \dot{+} T$, we can write $x=x_{1}+x_{2}$, where $x_{1} \in \mathcal{N}(A)$ and $x_{2} \in T$. Thus $y=A x=A x_{2} \in A T$. Therefore $\mathscr{R}(A) \subset A T$ and then $A T=\mathscr{R}(A)$.

$(3) \Rightarrow(1)$ Suppose that (3) is true, then by Theorem 6 , we know that $A_{T, S}^{(2, h)}=B$ exists, and then $\mathscr{R}(B)=T, \mathcal{N}(B)=S$. We need to show $A B A=A$ and $B$ is quasi-additive on $\mathscr{R}(A)$. Since $A_{T, S}^{(2, h)}=B$ exists, then $B A B=B$ and $B$ is quasi-additive on $A T$. Noting that for any $x \in X$, we have $A B A x \in A T$. Thus, $B(A B A-A) x=0$ and

$$
(A B A-A) x \subset \mathscr{R}(A) \cap \mathscr{N}(B)=\mathscr{R}(A) \cap S=\{0\}
$$

that is, $A B A=A$. Obviously, $A T \subset \mathscr{R}(A)$. On the converse, let $y \in \mathscr{R}(A)$. Since $Y=A T+S$, we have $y=y_{1}+y_{2}$ with some $y_{1} \in A T$ and $y_{2} \in S$. From $A T \subset \mathscr{R}(A)$, we see $y_{1} \in$ $\mathscr{R}(A)$. Thus, we have $y_{2}=y-y_{1} \in \mathscr{R}(A) \cap S=\{0\}$. So $\mathscr{R}(A) \subset A T$ and then $A T=\mathscr{R}(A)$. Hence, we obtain that $B$ is quasi-additive on $\mathscr{R}(A)$.

We should indicate that, for Definition 4, we define two different quasi-additivity in (iii) and (iv). But from Theorem 7 , we see that if the generalized inverse $A_{T, S}^{(h)}$ exists, then we must have $\mathscr{R}(A)=A T$. So there is no contradiction in our definition. The following proposition gives the uniqueness of $A_{T, S}^{(2, h)}$. From this result, it is also easy to see the uniqueness of $A_{T, S}^{(h)}$, which are similar to the linear (outer) generalized inverses with prescribed range and kernel.

Proposition 8. Let $A \in B(X, Y)$. Let $T$ and $S$ be homogeneous subsets in $X$ and $Y$, respectively. If the generalized inverse $A_{T, S}^{(2, h)}$ (or $A_{T, S}^{(h)}$ ) exists, then it is unique.

Proof. We only need to prove this proposition for $A_{T, S}^{(2, h)}$. By Definition 4, we need to prove that there is at most one bounded homogeneous operator $B \in H(Y, X)$ such that $B$ is quasi-additive on $A T$ and satisfies

$$
B A B=B, \quad \mathscr{R}(B)=T, \quad \mathcal{N}(B)=S .
$$

Suppose that there is another $C \in H(Y, X)$ such that $C$ is quasi-additive on $A T$ and satisfies the equations $C A C=C$, $\mathscr{R}(C)=T$, and $\mathscr{N}(C)=S$. Put $Q=A B$ and $Q^{\prime}=A C$. By using the equations $B A B=B$ and $C A C=C$, we see that 
both $Q$ and $Q^{\prime}$ are bounded homogeneous projectors on $Y$ and satisfy

$$
\mathscr{R}(Q)=\mathscr{R}\left(Q^{\prime}\right)=A T ; \quad \mathscr{N}(Q)=\mathscr{N}\left(Q^{\prime}\right)=S .
$$

Therefore, we get $Q=Q^{\prime}$. Thus, $C=C A C=C A B$. On the other hand, if we set $P=B A$ and $P^{\prime}=C A$, then by using the equations $B A B=B$ and $C A C=C$ again, and also noting that $B$ and $C$ are both quasi-additive on $A T$, similar to the proof $(1) \Rightarrow(2)$ in Theorem 6 , we see both $P$ and $P^{\prime}$ are bounded quasi-linear projectors and satisfy

$$
\mathscr{R}(P)=\mathscr{R}(B)=\mathscr{R}\left(P^{\prime}\right)=\mathscr{R}(C)=T .
$$

Thus, $B A=P=P^{\prime} P=C A B A$, and then $B=B A B=$ $C A B A B=C A B=C$.

Proposition 9. Let $A \in B(X, Y)$. Let $T$ and $S$ be homogeneous subsets in $X$ and $Y$, respectively. If the generalized inverse $A_{T, S}^{(2, h)}$ (or $A_{T, S}^{(h)}$ ) exists, then $T$ and $A T$ are both closed linear subspaces of $X$.

Proof. If the generalized inverse $A_{T, S}^{(2, h)}$ (or $A_{T, S}^{(h)}$ ) exists, then from Theorem 6 (or Theorem 7), we see $T$ is a closed linear subspace in $X$. Let $Q=A A_{T, S}^{(2, h)}$. Then it is easy to check that $\mathscr{R}(Q)=A T$. Since $A_{T, S}^{(2, h)}$ is quasi-additive on $A T$, simple computation can show that $Q=A A_{T, S}^{(2, h)}$ is a bounded quasilinear projector. Then from Lemma 3 , we get $A T$ is also a closed linear subspace of $X$ if $A_{T, S}^{(2, h)}$ exists. If the generalized inverse $A_{T, S}^{(h)}$ exists, then $A_{T, S}^{(2, h)}$ exists and $A_{T, S}^{(2, h)}=A_{T, S}^{(h)}$.

Finally, in this section, we will give some representation results of the generalized inverse $A_{T, S}^{(2, h)}$.

Theorem 10. Let $A \in B(X, Y)$. Let $T$ and $S$ be homogeneous subsets in $X$ and $Y$, respectively. Suppose that $G \in H(Y, X)$ such that $G$ is quasi-additive on $A T$, and $\mathscr{R}(G)=T, \mathcal{N}(G)=S$. Then the following statements are equivalent:

(1) the generalized inverse $A_{T, S}^{(2, h)}$ exists;

(2) $\left.(G A)\right|_{T}$ is invertible and $T \subset X$ is a closed linear subspace.

In this case, one has $A_{T, S}^{(2, h)}=\left(\left.(G A)\right|_{T}\right)^{-1} G$.

Proof. (1) $\Rightarrow$ (2) Suppose that (1) holds, then by Proposition 9, we get that $T \subset X$ is a closed linear subspace. If $G A x=0$ for some $x \in T$, then $x=G z$ for some $z \in Y$. Since $\mathcal{N}(G)=S$, by Theorem 6, we have $A x \in A T \cap S=\{0\}$. Noting that $\mathcal{N}(A) \cap$ $T=\{0\}$, thus, we get $x=0$ and then (2) holds.

(2) $\Rightarrow(1)$ Let $B=\left(\left.(G A)\right|_{T}\right)^{-1} G$. Obviously, we have $B A B=$ $B$, and $\mathscr{R}(B)=\mathscr{R}(G)=T, \mathcal{N}(B)=\mathcal{N}(G)=S$. We show that $B$ is quasi-additive on $A T$. Let $y \in Y$ and $z \in A T$. Since $G$ is quasi-additive on $A T$, we get that $\left(\left.(G A)\right|_{T}\right)^{-1}$ is a linear operator and then

$$
\begin{aligned}
B(y+z) & =\left(\left.(G A)\right|_{T}\right)^{-1} G(y+z) \\
& =\left(\left.(G A)\right|_{T}\right)^{-1}(G y+G z)=B y+B z .
\end{aligned}
$$

By Proposition 8 , the uniqueness of $A_{T, S}^{(2, h)}$, we can obtain $A_{T, S}^{(2, h)}=\left(\left.(G A)\right|_{T}\right)^{-1} G$.

Let $A \in B(X, Y)$. Recall that $A$ is group invertible if there exists $C \in B(Y, X)$ such that

$$
A C A=A, \quad C A C=C, \quad C A=A C .
$$

In this case, denote $C=A^{\#}$ as usual. For a bounded homogeneous operator $W \in H(X, Y)$, if there exists $W^{-} \epsilon$ $H(Y, X)$ such that $W W^{-} W=W$, then we call $W^{-}$as a bounded homogeneous inner inverse of $W$ or simply say $W$ is inner regular.

Theorem 11. Let $A \in B(X, Y)$. Let $T$ and $S$ be homogeneous subsets in $X$ and $Y$, respectively. Suppose that $G \in H(Y, X)$ such that $G$ is quasi-additive on $\mathscr{R}(A)$, and $\mathscr{R}(G)=\mathscr{R}(G A G)=T$, $\mathcal{N}(G)=S$. If $G A \in B(X, X)$ has the group inverse $(G A)^{\#}$, then $A_{T, S}^{(2, h)}$ exists and, moreover, $G A G \in H(Y, X)$ is inner regular and

$$
A_{T, S}^{(2, h)}=(G A)^{\#} G=G(G A G)^{-} G .
$$

Proof. Since $G$ is quasi-additive on $\mathscr{R}(A)$, we see $G A \in B(X)$ is well defined. Let $B=(G A)^{\#} G$, we show that $A_{T, S}^{(2, h)}=B$. From $\mathscr{R}(G)=\mathscr{R}(G A G)=T$, we get $\mathscr{R}(G A) \subset \mathscr{R}(G)=$ $\mathscr{R}(G A G) \subset \mathscr{R}(G A)$, and then $\mathscr{R}(G A)=\mathscr{R}(G)=T$. The existence of $(G A)^{\#}$ means that

$$
\mathscr{N}(G A) \cap \mathscr{R}(G A)=\{0\}, \quad G A\left(G A(G A)^{\#} G-G\right)=0 .
$$

Therefore

$$
\begin{gathered}
G A(G A)^{\#} G-G \in \mathcal{N}(G A) \cap \mathscr{R}(G) \\
=\mathscr{N}(G A) \cap \mathscr{R}(G A)=\{0\} .
\end{gathered}
$$

Hence $G=G A(G A)^{\#} G$. Now, we can compute as follows:

$$
\begin{aligned}
B A B & =(G A)^{\#} G A(G A)^{\#} G=(G A)^{\#} G=B, \\
T & =\mathscr{R}(G)=\mathscr{R}\left(G A(G A)^{\#} G\right) \\
& =\mathscr{R}\left((G A)^{\#} G A G\right) \subset \mathscr{R}\left((G A)^{\#} G\right) \\
& =\mathscr{R}(B)=\mathscr{R}\left((G A)^{\#} G A(G A)^{\#} G\right) \\
& =\mathscr{R}\left(G A\left((G A)^{\#}\right)^{2} G\right) \subset \mathscr{R}(G) \\
& =T, \\
S & =\mathscr{N}(G) \subset \mathcal{N}\left((G A)^{\#} G\right) \\
& =\mathscr{N}(B) \subset \mathcal{N}\left(G A(G A)^{\#} G\right)=\mathscr{N}(G) \\
= & S .
\end{aligned}
$$

Thus, $\mathscr{R}(B)=T$ and $\mathscr{N}(B)=S$. Obviously, we see $B$ is quasi-additive on $A T$ for $A T \subset \mathscr{R}(A)$. So, from Definition 4 and Proposition 8, we see $A_{T, S}^{(2, h)}$ exists and $A_{T, S}^{(2, h)}=(G A)^{\#} G$. 
Set $C=A\left((G A)^{\#}\right)^{2}$. Then it is easy to check that

$(G A G) C(G A G)=(G A G) A\left((G A)^{\#}\right)^{2}(G A G)=G A G$.

We get $G A G$ is inner regular and $(G A G)^{-}=A\left((G A)^{\#}\right)^{2}$. Furthermore, we have

$$
G(G A G)^{-} G=G C G=G A\left((G A)^{\#}\right)^{2} G=(G A)^{\#} G=A_{T, S}^{(2, h)} .
$$

This completes the proof.

Theorem 12. Let $A \in B(X, Y)$. Let $T$ and $S$ be homogeneous subsets in $X$ and $Y$, respectively. Suppose that $G \in H(Y, X)$ such that $G$ is quasi-additive on $\mathscr{R}(A)$, and $\mathscr{R}(G)=\mathscr{R}(G A G)=T$, $\mathcal{N}(G)=S$. If $G A \in B(X)$ has the group inverse $(G A)^{\#}$, then $A_{T, S}^{(2, h)}$ exists and $A_{T, S}^{(2, h)}=\lim _{\lambda \rightarrow 0, \lambda \notin \sigma(-G A)}\left(\lambda I_{X}+G A\right)^{-1} G$.

Proof. By Theorem 11, we know that $A_{T, S}^{(2, h)}$ exists and $A_{T, S}^{(2, h)}=$ $(G A)^{\#} G$. Let $P=A_{T, S}^{(2, h)} A$. Noting that $G$ is quasi-additive on $\mathscr{R}(A)$, then $P \in B(X)$ is a projector and $P=(G A)^{\#} G A$. Clearly, we see that $G A,(G A)^{\#} \in B(\mathscr{R}(P))$ and $G A$ is invertible in $B(\mathscr{R}(P))$ with $\left(\left.G A\right|_{B(\mathscr{R}(P))}\right)^{-1}=(G A)^{\#}$. Consider the decomposition $X=\mathscr{R}(P) \oplus \mathscr{R}\left(I_{X}-P\right)$. Then we can write $\lambda I_{X}+G A$ as the following matrix form:

$$
\lambda I_{X}+G A=\left[\begin{array}{ll}
\lambda P+G A & \\
& \lambda\left(I_{X}-P\right)
\end{array}\right] .
$$

From (23), we see if $\lambda \notin \sigma(-G A) \cup\{0\}$, then $\lambda P+G A$ is invertible in $B(\mathscr{R}(P))$. Thus, in the case, by (23) we have

$$
\left(\lambda I_{X}+G A\right)^{-1}=\left[\begin{array}{ll}
(\lambda P+G A)^{-1} & \\
& \lambda^{-1}\left(I_{X}-P\right)
\end{array}\right] .
$$

From the proof of Theorem 11, we know that $\mathscr{R}(G A)=$ $\mathscr{R}(G)=T$. Thus, we get $\mathcal{N}\left(I_{X}-P\right)=\mathscr{R}(P)=\mathscr{R}\left((G A)^{\#} G A\right)=$ $T$. So, by using (24), we can obtain that $\left(\lambda I_{X}+G A\right)^{-1} G=$ $P(\lambda P+G A)^{-1} G$. Now we can compute as follows:

$$
\begin{aligned}
A_{T, S}^{(2, h)} & =P(G A)^{\#} G \\
& =\lim _{\substack{\lambda \rightarrow 0 \\
\lambda \notin \sigma(-G A)}} P(\lambda P+G A)^{-1} G \\
& =\lim _{\substack{\lambda \rightarrow 0 \\
\lambda \notin \sigma(-G A)}}\left(\lambda I_{X}+G A\right)^{-1} G .
\end{aligned}
$$

This completes the proof.

\section{Perturbations of the Generalized Inverses $A_{T, S}^{(2, h)}$ and $A_{T, S}^{(h)}$}

In this section, we present some perturbation results for the generalized inverse $A_{T, S}^{(2, h)}$ and $A_{T, S}^{(h)}$. The next lemma, which is a generalized Neumman Lemma taken from [19], will be useful for us to improve perturbation bound.
Lemma 13 (see [19]). Let $X$ be a Banach space and $P \in B(X)$. If there exist two constants $\lambda_{1}, \lambda_{2} \in[0,1)$ such that

$$
\|P x\| \leq \lambda_{1}\|x\|+\lambda_{2}\|(I+P) x\|, \quad(\forall x \in X),
$$

then $I+P: X \rightarrow X$ is bijective. Moreover, for any $x \in X$

$$
\begin{aligned}
& \frac{1-\lambda_{1}}{1+\lambda_{2}}\|x\| \leq\|(I+P) x\| \leq \frac{1+\lambda_{1}}{1-\lambda_{2}}\|x\|, \\
& \frac{1-\lambda_{2}}{1+\lambda_{1}}\|x\| \leq\left\|(I+P)^{-1} x\right\| \leq \frac{1+\lambda_{2}}{1-\lambda_{1}}\|x\| .
\end{aligned}
$$

Let $A, \delta A \in B(X, Y)$. Suppose that $T \subset X$ and $S \subset Y$ are homogeneous subsets such that $A_{T, S}^{(2, h)}$ exists. If $A_{T, S}^{(2, h)}$ is quasiadditive on $\mathscr{R}(\delta A)$, then we see that $A_{T, S}^{(2, h)} \delta A$ is a bounded linear operator, so $I_{X}+A_{T, S}^{(2, h)} \delta A$ is also a bounded linear operator on $X$. Furthermore, if $A_{T, S}^{(2, h)}$ and $\delta A$ also satisfy

$$
\begin{aligned}
& \left\|A_{T, S}^{(2, h)} \delta A x\right\| \leq \lambda_{1}\|x\| \\
& +\lambda_{2}\left\|\left(I_{X}+A_{T, S}^{(2, h)} \delta A\right) x\right\|, \quad(\forall x \in X),
\end{aligned}
$$

where $\lambda_{1}, \lambda_{2} \in(-1,1)$, then by Lemma 13, we get that $I_{X}+$ $A_{T, S}^{(2, h)} \delta A$ is invertible.

Lemma 14. Let $A \in H(X, Y)$ and $B \in H(Y, X)$ such that $A$ is quasi-additive on $\mathscr{R}(B)$ and $B$ is quasi-additive on $\mathscr{R}(A)$; then $I_{Y}+A B$ is invertible in $H(Y, Y)$ if and only if $I_{X}+B A$ is invertible in $H(X, X)$.

Proof. If there is a $\Phi \in H(Y, Y)$ such that $\left(I_{Y}+A B\right) \Phi=\Phi\left(I_{Y}+\right.$ $A B)=I_{Y}$, then

$$
\begin{aligned}
I_{X} & =I_{X}+B A-B A=I_{X}+B A-S\left(\left(I_{Y}+A B\right) \Phi\right) A \\
& =I_{X}+B A-((B+B A B) \Phi) A
\end{aligned}
$$

(B quasi-additive on $\mathscr{R}(A)$ )

$$
\begin{aligned}
& =I_{X}+B A-\left(\left(I_{X}+B A\right) S \Phi\right) A \\
& =\left(I_{X}+B A\right)\left(1_{X}-B \Phi A\right)
\end{aligned}
$$

(A quasi-additive on $\mathscr{R}(B))$.

Similarly, we also have $I_{X}=\left(I_{X}-B \Phi A\right)\left(I_{X}+B A\right)$. Thus, $I_{X}+B A$ is invertible on $X$ with $\left(I_{X}+B A\right)^{-1}=\left(1_{X}-B \Phi A\right) \epsilon$ $H(X, X)$.

The converse can also be proved by using the same way as above.

Lemma 15. Let $A, \delta A \in B(X, Y)$. Suppose that $T \subset X$ and $S \subset$ $Y$ are homogeneous subsets such that $A_{T, S}^{(2, h)}$ exists. If $A_{T, S}^{(2, h)}$ is quasi-additive on $\mathscr{R}(\delta A)$ and $I_{Y}+\delta A A_{T, S}^{(2, h)}$ (resp., $I_{X}+A_{T, S}^{(2, h)} \delta A$ ) is invertible in $H(Y, Y)$ (resp., $H(X, X)$ ), then $I_{X}+A_{T, S}^{(2, h)} \delta A$ (resp., $\left.I_{Y}+\delta A A_{T, S}^{(2, h)}\right)$ is invertible in $H(X, X)$ (resp., $H(Y, Y)$ ) and

$$
\Phi=A_{T, S}^{(2, h)}\left(I_{Y}+\delta A A_{T, S}^{(2, h)}\right)^{-1}=\left(I_{X}+A_{T, S}^{(2, h)} \delta T\right)^{-1} A_{T, S}^{(2, h)}
$$

is a bounded homogeneous operator with $\mathscr{R}(\Phi)=T$ and $\mathcal{N}(\Phi)=S$. 
Proof. By Lemma 14, we obtain that $I_{Y}+\delta A A_{T, S}^{(2, h)}$ (resp., $I_{X}+$ $A_{T, S}^{(2, h)} \delta A$ ) is invertible in $H(Y, Y)$ (resp., $H(X, X)$ ).

Clearly, $I_{X}+A_{T, S}^{(2, h)} \delta A$ is a bounded linear operator and $I_{Y}+\delta A A_{T, S}^{(2, h)} \in H(Y, Y)$. From the equation

$$
\left(I_{X}+A_{T, S}^{(2, h)} \delta A\right) A_{T, S}^{(2, h)}=A_{T, S}^{(2, h)}\left(I_{Y}+\delta A A_{T, S}^{(2, h)}\right)
$$

and $A_{T, S}^{(2, h)} \in H(Y, X)$, we get that $\Phi$ is a bounded homogeneous operator. Finally, by using (30) and Definition 4 , we can obtain that $\mathscr{R}(\Phi)=\mathscr{R}\left(A_{T, S}^{(2, h)}\right)=T$ and $\mathscr{N}(\Phi)=\mathscr{N}\left(A_{T, S}^{(2, h)}\right)=$ $S$.

Theorem 16. Let $A, \delta A \in B(X, Y)$. Suppose that $T \subset X$ and $S \subset Y$ are homogeneous subsets such that $A_{T, S}^{(2, h)}$ exists. Put $\bar{A}=$ $A+\delta A$. If $A_{T, S}^{(2, h)}$ is quasi-additive on $\mathscr{R}(\delta A)$ and satisfies (28), then $\bar{A}_{T, S}^{(2, h)}$ exists and $\bar{A}_{T, S}^{(2, h)}=\left(I_{X}+A_{T, S}^{(2, h)} \delta A\right)^{-1} A_{T, S}^{(2, h)}$. Moreover, one has

$$
\frac{\left\|\bar{A}_{T, S}^{(2, h)}-A_{T, S}^{(2, h)}\right\|}{\left\|A_{T, S}^{(2, h)}\right\|} \leq \frac{1+\lambda_{2}}{1-\lambda_{1}}\left\|A_{T, S}^{(2, h)} \delta A\right\| .
$$

Proof. Since $A_{T, S}^{(2, h)}$ is quasi-additive on $\mathscr{R}(\delta A)$ and satisfies (28), then from Lemma 13 and Lemma 15 , we see $\Phi=$ $A_{T, S}^{(2, h)}\left(I_{Y}+\delta A A_{T, S}^{(2, h)}\right)^{-1}=\left(I_{X}+A_{T, S}^{(2, h)} \delta T\right)^{-1} A_{T, S}^{(2, h)}$ is well defined with $\mathscr{R}(\Phi)=T$ and $\mathcal{N}(\Phi)=S$. Now, we prove that $\Phi$ is quasiadditive on $\bar{A} T$. Let $x \in X$ and $z \in \bar{A} T$, then $z=\bar{A} w=A w+$ $\delta A w$ for some $w \in T$. Since $A_{T, S}^{(2, h)}$ is quasi-additive on $\mathscr{R}(\delta A)$ and $A T$, also noting that $I_{X}+A_{T, S}^{(2, h)} \delta A$ is a bounded linear operator, then

$$
\begin{aligned}
\Phi(x+z)= & \left(I_{X}+A_{T, S}^{(2, h)} \delta T\right)^{-1} A_{T, S}^{(2, h)}(x+A w+\delta A w) \\
= & \left(I_{X}+A_{T, S}^{(2, h)} \delta T\right)^{-1} A_{T, S}^{(2, h)}(x+A w) \\
& +\left(I_{X}+A_{T, S}^{(2, h)} \delta T\right)^{-1} A_{T, S}^{(2, h)} \delta A w \\
= & \Phi x+\Phi A w+\Phi \delta A w \\
= & \Phi x+\Phi z .
\end{aligned}
$$

We get $\Phi$ is quasi-additive on $\bar{A} T$. Thus, from Definition 4 and Proposition 8 , we see that $\bar{A}_{T, S}^{(2, h)}$ exists and $\bar{A}_{T, S}^{(2, h)}=\left(I_{X}+\right.$ $\left.A_{T, S}^{(2, h)} \delta A\right)^{-1} A_{T, S}^{(2, h)}$.

Finally, we show that the estimate formula is valid:

$$
\begin{aligned}
& \frac{\left\|\bar{A}_{T, S}^{(2, h)}-A_{T, S}^{(2, h)}\right\|}{\left\|A_{T, S}^{(2, h)}\right\|} \\
& \leq \frac{\left\|\left(I_{X}+A_{T, S}^{(2, h)} \delta T\right)^{-1} A_{T, S}^{(2, h)}-A_{T, S}^{(2, h)}\right\|}{\left\|A_{T, S}^{(2, h)}\right\|} \\
& \leq \frac{\left\|\left(I_{X}+A_{T, S}^{(2, h)} \delta T\right)^{-1}\left[I_{X}-\left(I_{X}+A_{T, S}^{(2, h)} \delta T\right)\right] A_{T, S}^{(2, h)}\right\|}{\left\|A_{T, S}^{(2, h)}\right\|} \\
& \leq\left\|\left(I_{X}+A_{T, S}^{(2, h)} \delta T\right)^{-1}\right\|\left\|A_{T, S}^{(2, h)} \delta A\right\| .
\end{aligned}
$$

By using Lemma 13, we get

$$
\left\|\left(I_{X}+T^{H} \delta T\right)^{-1}\right\| \leq \frac{1+\lambda_{2}}{1-\lambda_{1}} .
$$

Thus from (34) and (35), we can obtain

$$
\frac{\left\|\bar{A}_{T, S}^{(2, h)}-A_{T, S}^{(2, h)}\right\|}{\left\|A_{T, S}^{(2, h)}\right\|} \leq \frac{1+\lambda_{2}}{1-\lambda_{1}}\left\|A_{T, S}^{(2, h)} \delta A\right\| .
$$

This completes the proof.

Corollary 17. Let $A, \delta A \in B(X, Y)$. Suppose that $T \subset X$ and $S \subset Y$ are homogeneous subsets such that $A_{T, S}^{(2, h)}$ exists. Put $\bar{A}=$ $A+\delta A$. If $A_{T, S}^{(2, h)}$ is quasi-additive on $\mathscr{R}(\delta A)$ with $\left\|A_{T, S}^{(2, h)} \delta A\right\|<$ 1 , then $\bar{A}_{T, S}^{(2, h)}$ exists and $\bar{A}_{T, S}^{(2, h)}=\left(I_{X}+A_{T, S}^{(2, h)} \delta A\right)^{-1} A_{T, S}^{(2, h)}$. Moreover, one has

$$
\frac{\left\|\bar{A}_{T, S}^{(2, h)}-A_{T, S}^{(2, h)}\right\|}{\left\|A_{T, S}^{(2, h)}\right\|} \leq \frac{\left\|A_{T, S}^{(2, h)} \delta T\right\|}{1-\left\|A_{T, S}^{(2, h)} \delta A\right\|} .
$$

Proof. Obviously, this corollary follows from Theorem 16 if we take $\lambda_{1}=\left\|A_{T, S}^{(2, h)} \delta T\right\|$ and $\lambda_{2}=0$.

Lemma 18 (Neumann Lemma for quasi-linear operators]). Let $A \in H(X, X)$. If $A$ is quasi-additive on $\mathscr{R}(A)$ with $\|A\|<$ 1 , then $(I+A)^{-1}$ exists and

$$
(I+A)^{-1}=\sum_{n=0}^{\infty}(-A)^{n}, \quad\left\|(I+A)^{-1}\right\| \leq \frac{1}{1-\|A\|} .
$$

Proof. Noting that $A$ is quasi-additive on $\mathscr{R}(A)$, then the proof is almost the same to the classical Neumann Lemma for bounded linear operators. Here we omit it, and we refer to [20, Lemma 4.2.6] for more information.

Theorem 19. Let $A, \delta A \in B(X, Y)$. Suppose that $T \subset X$ and $S \subset Y$ are homogeneous subsets such that $A_{T, S}^{(2, h)}$ exists. Put $\bar{A}=$ $A+\delta A$. If $A_{T, S}^{(2, h)}$ is quasi-additive on $\mathscr{R}(\delta A)$ with $\left\|\delta A A_{T, S}^{(2, h)}\right\|<$ 1 , then

(1) $I_{Y}+\delta A A_{T, S}^{(2, h)}$ is inventible bounded homogeneous operator;

(2) $\bar{A}_{T, S}^{(2, h)}$ exists and $\bar{A}_{T, S}^{(2, h)}=A_{T, S}^{(2, h)}\left(I_{Y}+\delta A A_{T, S}^{(2, h)}\right)^{-1}$.

Moreover, one has

$$
\frac{\left\|\bar{A}_{T, S}^{(2, h)}-A_{T, S}^{(2, h)}\right\|}{\left\|A_{T, S}^{(2, h)}\right\|} \leq \frac{\left\|\delta A A_{T, S}^{(2, h)}\right\|}{1-\left\|\delta A A_{T, S}^{(2, h)}\right\|} .
$$

Proof. Since $A_{T, S}^{(2, h)}$ is quasi-additive on $\mathscr{R}(\delta A)$, then $\mathscr{R}\left(\delta A A_{T, S}^{(2, h)}\right) \subset \mathscr{R}(\delta A)$ implies that $A_{T, S}^{(2, h)}$ is quasi-additive on $\mathscr{R}\left(\delta A A_{T, S}^{(2, h)}\right)$. Obviously, $\delta A A_{T, S}^{(2, h)} \in H(Y)$, and it follows from Lemma 18 that $I_{Y}+\delta A A_{T, S}^{(2, h)}$ is inventible bounded homogeneous operator. Then, by using Lemma 15 , similar to 
the proof of Theorem 16, we can show that $\bar{A}_{T, S}^{(2, h)}$ exists and $\bar{A}_{T, S}^{(2, h)}=A_{T, S}^{(2, h)}\left(I_{Y}+\delta A A_{T, S}^{(2, h)}\right)^{-1}$. For the estimate formula, we can compute that

$$
\begin{aligned}
& \frac{\left\|\bar{A}_{T, S}^{(2, h)}-A_{T, S}^{(2, h)}\right\|}{\left\|A_{T, S}^{(2, h)}\right\|} \\
& \leq \frac{\left\|A_{T, S}^{(2, h)}\left(I_{Y}+\delta A A_{T, S}^{(2, h)}\right)^{-1}-A_{T, S}^{(2, h)}\right\|}{\left\|A_{T, S}^{(2, h)}\right\|} \\
& =\frac{\left\|\left[A_{T, S}^{(2, h)}-A_{T, S}^{(2, h)}\left(I_{Y}+\delta A A_{T, S}^{(2, h)}\right)\right]\left(I_{Y}+\delta A A_{T, S}^{(2, h)}\right)^{-1}\right\|}{\left\|A_{T, S}^{(2, h)}\right\|} \\
& \leq\left\|\delta A A_{T, S}^{(2, h)}\right\|\left\|\left(I_{Y}+\delta A A_{T, S}^{(2, h)}\right)^{-1}\right\| .
\end{aligned}
$$

Thus, our result follows from Lemma 18 and (40).

Finally, we present two perturbation results for the generalized inverse $A_{T, S}^{(h)}$.

Theorem 20. Let $A, \delta A \in B(X, Y)$. Suppose that $T \subset X$ and $S \subset Y$ are homogeneous subsets such that $A_{T, S}^{(2, h)}$ exists. Put $\bar{A}=$ $A+\delta A$. If $A_{T, S}^{(2, h)}$ is quasi-additive on $\mathscr{R}(\delta A)$ and $I_{X}+A_{T, S}^{(2, h)} \delta A$ is invertible in $H(X, X)$, then $\bar{A}_{T, S}^{(h)}=\left(I_{X}+A_{T, S}^{(2, h)} \delta A\right)^{-1} A_{T, S}^{(2, h)}$ if and only if $\mathscr{R}(\bar{A}) \cap S=\{0\}$.

Proof. If $\bar{A}_{T, S}^{(h)}=\left(I_{X}+A_{T, S}^{(2, h)} \delta A\right)^{-1} A_{T, S}^{(2, h)}$, then from Theorem 7 , obviously, we have $\mathscr{R}(\bar{A}) \cap S=\{0\}$. Conversely, suppose that $\mathscr{R}(\bar{A}) \cap S=\{0\}$. First, noting that from Lemma 15, we get $\Phi=\left(I_{X}+A_{T, S}^{(2, h)} \delta A\right)^{-1} A_{T, S}^{(2, h)}$ is well defined, and $\mathscr{R}(\Phi)=T$ and $\mathcal{N}(\Phi)=S$. Similar to the proof of Theorem 16, we can show $\bar{A}_{T, S}^{(2, h)}$ exists and $\bar{A}_{T, S}^{(2, h)}=\Phi$. Noting that $\bar{A}_{T, S}^{(h)}$ is unique if it exists, thus, by using Theorems 6 and 7, we can prove our theorem.

Corollary 21. Let $A, \delta A \in B(X, Y)$. Suppose that $T \subset X$ and $S \subset Y$ are homogeneous subsets such that $A_{T, S}^{(2, h)}$ exists. Put $\bar{A}=$ $A+\delta A$. If $A_{T, S}^{(2, h)}$ is quasi-additive on $\mathscr{R}(\delta A)$ with $\left\|A_{T, S}^{(2, h)} \delta A\right\|<$ 1, then $\bar{A}_{T, S}^{(h)}=\left(I_{X}+A_{T, S}^{(2, h)} \delta A\right)^{-1} A_{T, S}^{(2, h)}$ if and only if $\mathscr{R}(\bar{A}) \cap S=$ $\{0\}$.

Proof. By our assumption, $A_{T, S}^{(2, h)}$ is quasi-additive on $\mathscr{R}(\delta A)$, we get $I_{X}+A_{T, S}^{(2, h)} \delta A$ is a bounded linear operator, and then it is well known that $I_{X}+A_{T, S}^{(2, h)} \delta A$ is invertible when $\left\|A_{\mathrm{T}, S}^{(2, h)} \delta A\right\|<$ 1. So the corollary follows from Theorem 20.

\section{Acknowledgment}

The authors thank the referees for their helpful comments and suggestions.

\section{References}

[1] X. Y. Bai, Y. W. Wang, G. Q. Liu, and J. Xia, "Definition and criterion for a homogeneous generalized inverse," Acta Mathematica Sinica, vol. 52, no. 2, pp. 353-360, 2009.

[2] Y. Wang and S. Pan, "An approximation problem of the finite rank operator in Banach spaces," Science in China A, vol. 46, no. 2, pp. 245-250, 2003.

[3] Y. Wang, Generalized Inverse of Operator in Banach Spaces and Applications, Science Press, Beijing, China, 2005.

[4] A. Ben-Israel and T. N. E. Greville, Generalized Inverses: Theory and Applications, Springer, New York, NY, USA, 2nd edition, 2003.

[5] Y. Xue, Stable Perturbations of Operators and Related Topics, World Scientific Publishing, 2012.

[6] D. S. Djordjević and P. S. Stanimirović, "On the generalized Drazin inverse and generalized resolvent," Czechoslovak Mathematical Journal, vol. 51, no. 3, pp. 617-634, 2001.

[7] D. S. Djordjević and Y. Wei, "Outer generalized inverses in rings," Communications in Algebra, vol. 33, no. 9, pp. 3051-3060, 2005.

[8] F. Du and Y. Xue, "Perturbation analysis of $A_{T, S}^{(2)}$ on Banach spaces," Electronic Journal of Linear Algebra, vol. 23, pp. 586598, 2012.

[9] Y. Wei, "A characterization and representation of the generalized inverse $A_{T, S}^{(2)}$ and its applications," Linear Algebra and Its Applications, vol. 280, no. 2-3, pp. 87-96, 1998.

[10] Y. Wei and H. Wu, "The representation and approximation for the generalized inverse $A_{T, S}^{(2)}$," Applied Mathematics and Computation, vol. 135, no. 2-3, pp. 263-276, 2003.

[11] Y. Wei and H. Wu, "On the perturbation and subproper splittings for the generalized inverse $A_{T, S}^{(2)}$ of rectangular matrix $A$," Journal of Computational and Applied Mathematics, vol. 137, no. 2, pp. 317-329, 2001.

[12] Y. Yu and G. Wang, "The generalized inverse $A_{T, S}^{(2)}$ over commutative rings," Linear and Multilinear Algebra, vol. 53, no. 4, pp. 293-302, 2005.

[13] J. Cao and Y. Xue, "Perturbation analysis of bounded homogeneousgeneralized inverses on Banach spaces," arXiv preprint, http://arxiv.org/abs/1302.3965.

[14] J. Cao and Y. Xue, "On the simplest expression of the perturbed Moore-Penrose metric generalized inverse," Preprint.

[15] P. Liu and Y. W. Wang, "The best generalised inverse of the linear operator in normed linear space," Linear Algebra and Its Applications, vol. 420, no. 1, pp. 9-19, 2007.

[16] M. Z. Nashed and G. F. Votruba, "A unified operator theory of generalized inverses," in Generalized Inverses and Applications, M. Z. Nashed, Ed., pp. 1-109, Academic Press, New York, NY, USA, 1976.

[17] R. X. Ni, "Moore-Penrose metric generalized inverses of linear operators in arbitrary Banach spaces," Acta Mathematica Sinica, vol. 49, no. 6, pp. 1247-1252, 2006.

[18] H. Wang and Y. Wang, "Metric generalized inverse of linear operator in Banach space," Chinese Annals of Mathematics B, vol. 24, no. 4, pp. 509-520, 2003.

[19] J. Ding, "New perturbation results on pseudo-inverses of linear operators in Banach spaces," Linear Algebra and Its Applications, vol. 362, pp. 229-235, 2003.

[20] H. Ma, Construction of some generalized inverses of operators between Banach spaces and their selections, perturbations and applications [Ph.D. thesis], 2012. 


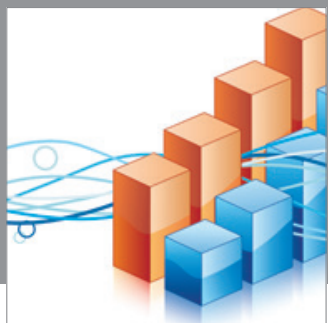

Advances in

Operations Research

mansans

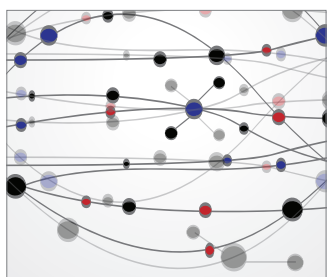

The Scientific World Journal
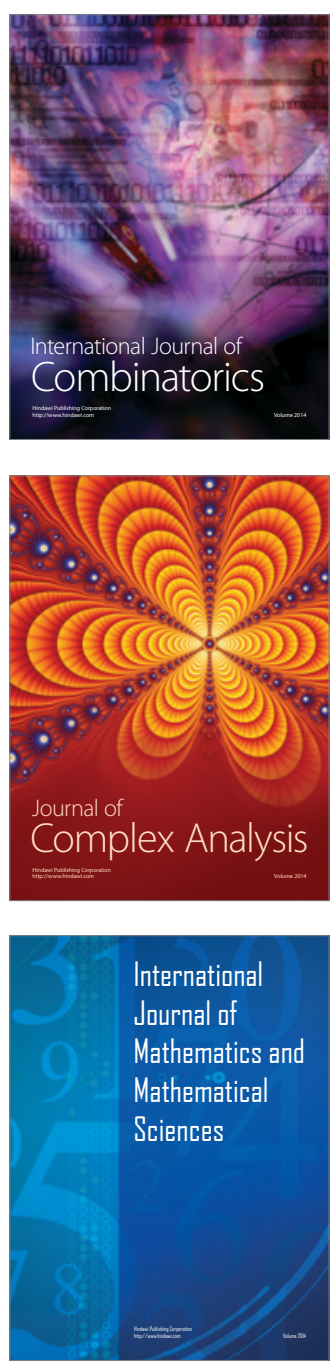
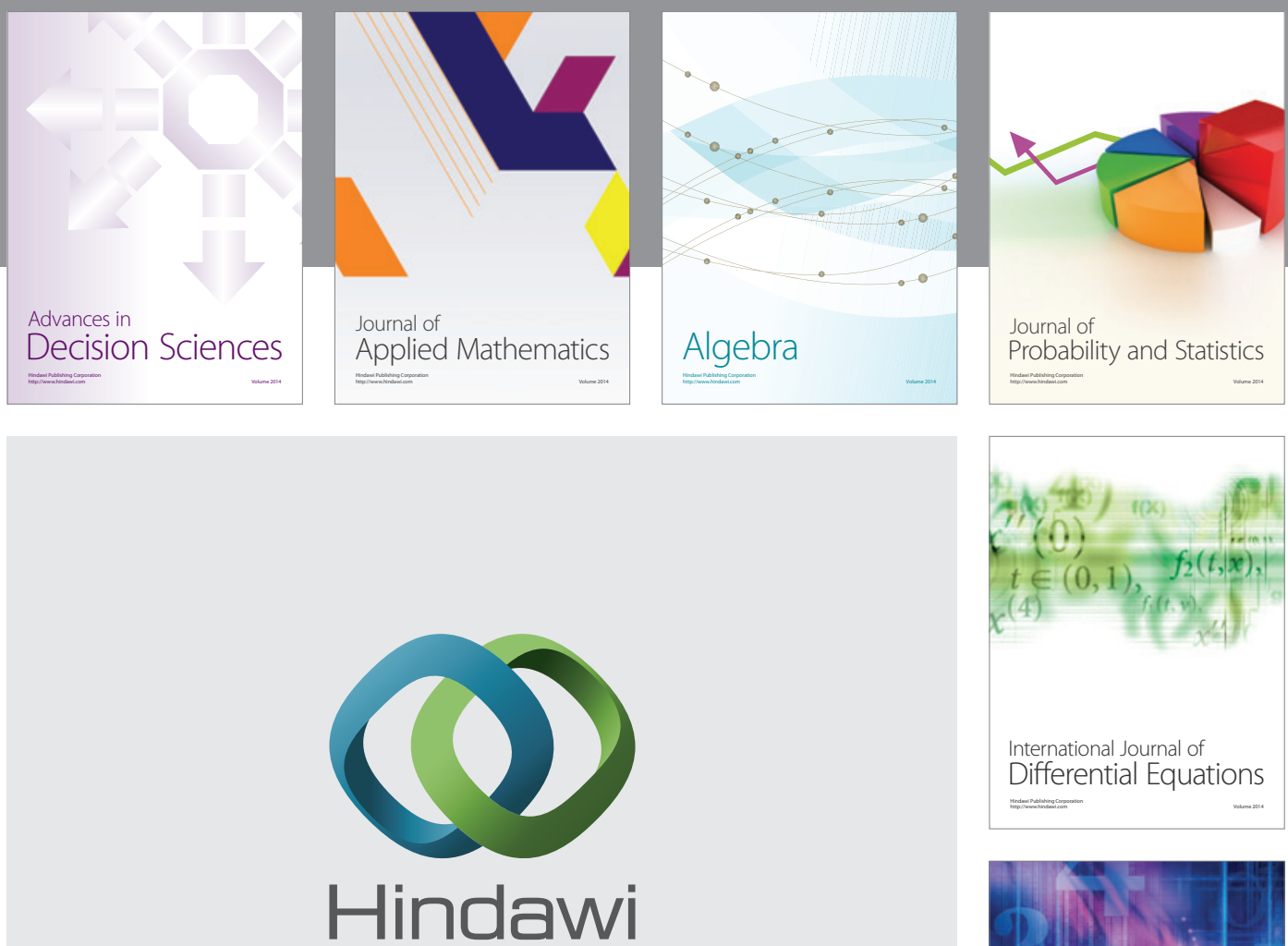

Submit your manuscripts at http://www.hindawi.com
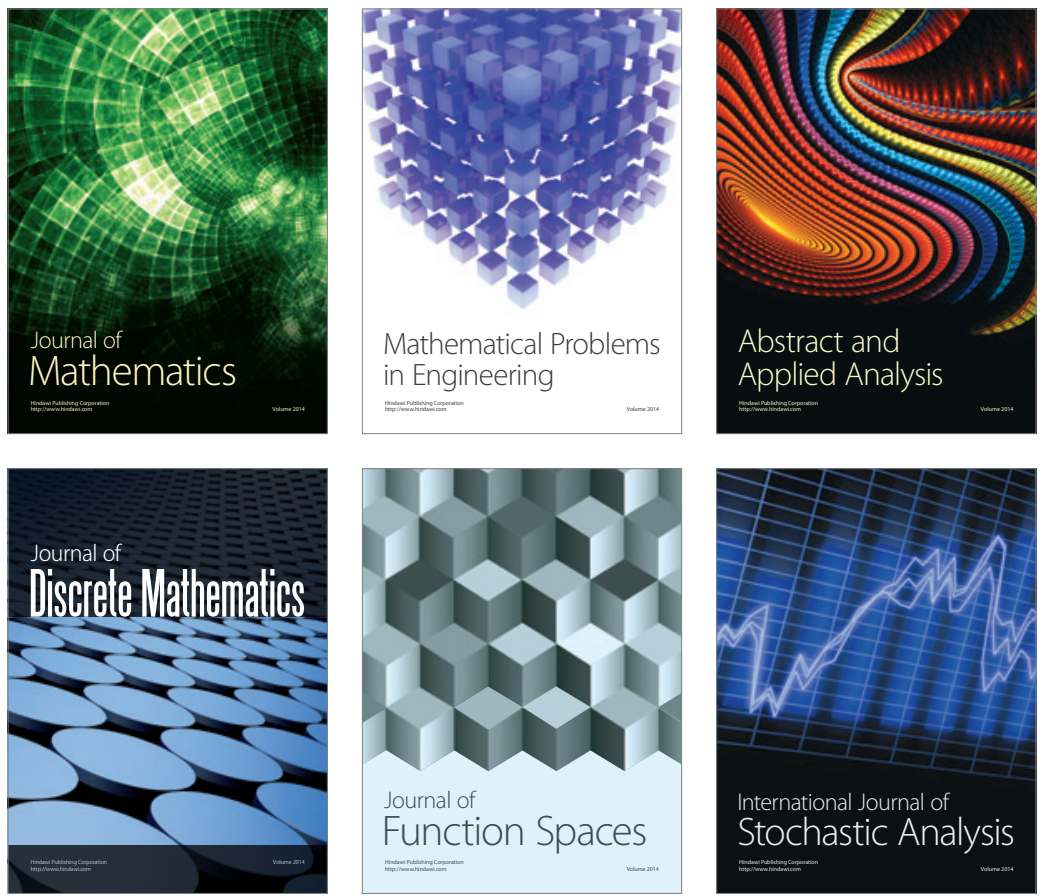

Journal of

Function Spaces

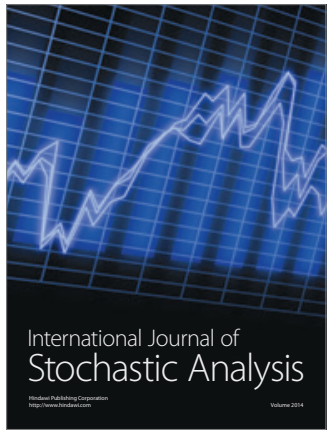

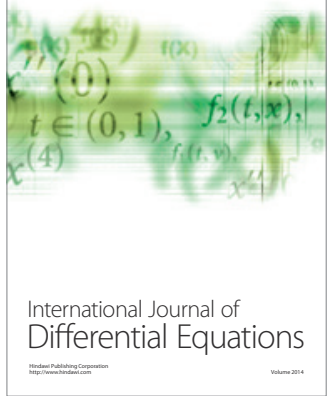
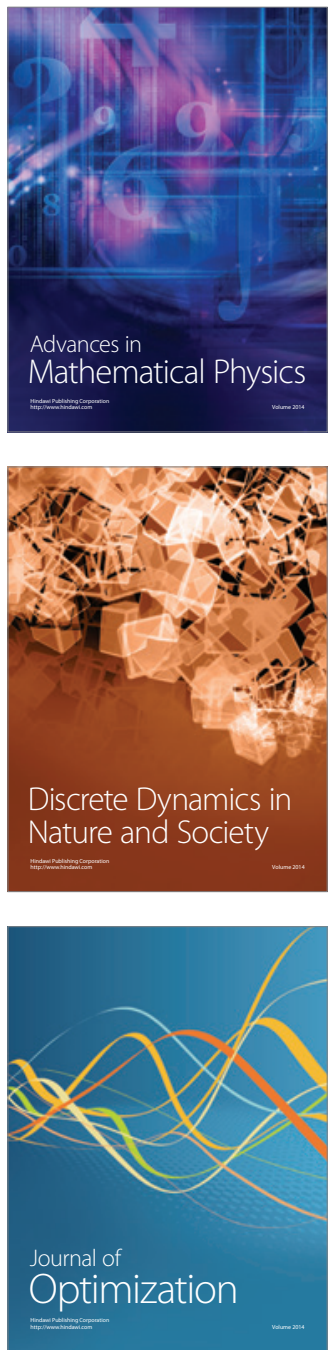